\title{
A Vertical Handover Algorithm in Integrated Macrocell Femtocell Networks
}

\author{
Suman Deswal, Anita Singhrova \\ Computer Science \& Engineering Department, Deenbandhu Chhotu Ram University of Science \& Technology, \\ Murthal, India
}

\begin{tabular}{l} 
Article Info \\
\hline Article history: \\
Received Jul 28, 2016 \\
Revised Oct 10, 2016 \\
Accepted Oct 25, 2016 \\
\hline
\end{tabular}

Keyword:

Femtocell

Handover

Macrocell

Packet loss ratio

Received signal strength

\begin{abstract}
The explosion in wireless telecommunication technologies has lead to a huge increase in the number of mobile users. The greater dependency on the mobile devices has raised the user's expectations to always remain best connected. In the process, the user is always desiringgood signal strength even at certain black spots and indoors. Moreover, the exponential growth of the number of mobile devices has overloaded macrocells. Femtocells have emerged out as a good promising solution for complete coverage indoors and for offloading macrocell. Therefore, a new handover strategy between femtocells and macrocell is proposed in this paper. The proposed handover algorithm is mainly based on calculating equivalent received signal strength along with dynamic margin for performing handover. The simulation results of proposed algorithm are compared with the traditional algorithm. The proposed strategy shows improvement in two major performance parameters namely reduction in unnecessary handovers and Packet Loss Ratio. The quantitative analysis further shows $55.27 \%$ and $23.03 \%$ reduction in packet loss ratio and $61.85 \%$ and $36.78 \%$ reduction in unnecessary handovers at a speed of $120 \mathrm{kmph}$ and $30 \mathrm{kmph}$ respectively. Moreover, the proposed algorithm proves to be an efficient solution for both slow and fast moving vehicles.
\end{abstract}

Copyright $@ 2017$ Institute of Advanced Engineering and Science. All rights reserved.

\section{Corresponding Author:}

Suman Deswal,

Computer Science \& Engineering Department,

Deenbandhu Chhotu Ram University of Science \& Technology, Murthal, India.

Email: suman_gulia2000@yahoo.co.in

\section{INTRODUCTION}

The Long Term Evolution system (LTE) is wireless communication standard for high speed data. This standard is developed by 3GPP (Third Generation Partnership Project), which aimed to make a globally applicable third generation mobile system specification. The 3GPP includes the development of Enhanced Data for Global Evolution (EDGE), General Packet Radio Service (GPRS), High Speed Packet Access (HSPA), LTE and 4G networks. LTE is a packet switched network used for both real time applications and data and is also termed as Evolved Universal Terrestrial Access Network (E-UTRAN). It came into existence with the fast development in wireless communications and increase in the demand for higher data rates and quality of service. Based on Orthogonal Frequency Division Multiple Access (OFDMA) and a bandwidth of as large as $20 \mathrm{MHz}$, layered OFDMA radio access scheme is used to achieve high data rate in LTEAdvanced [1]. LTE provides high data rates as high as $100 \mathrm{Mbps}$ in downlink and $75 \mathrm{Mbps}$ in uplink. The LTE network consists of macrocell base stations termed as eNB, evolved nodeB. The base stations are connected together with the help of X2 interface and to the core network with the help of S1 interface as shown in Figure 1 [2]. The core network consists of Mobility Management Entity (MME) which provides the functions related to establishment of connection between the device and the network. The second important 
element of core network is Serving Gateway (S-GW) which acts as an interface between the packet data network and eNB [3].

Even after operating at a high bandwidth providing high data rates, full coverage is not provided indoors in such networks. Moreover, due to an increase in the number of users in today's scenario and due to many video streaming applications, the overall traffic in eNB is increased. The user located far away from macrocell base station suffers from poor capacity due to higher pass loss [4].

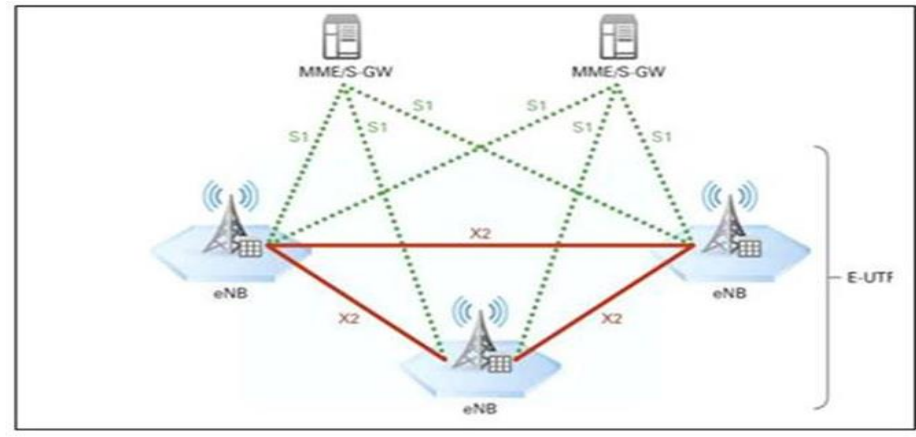

Figure 1. Architecture of LTE Networks

Femtocells are introduced to provide complete coverage. The femtocells are plugandplay, low power, low cost devices which operate in licensed indoorspectrum and have a very short range of upto 30 meters only. They connect the mobile devices to the core network of mobile operator through the broadband connection and improve the capacity and coverage of cellular networks [5]. They support limited simultaneous users in indoor environment [6]. Femtocells are overlaid on macrocells and as the device moves across a macrocell overlaid with femtocells, the mobility management becomes an integral part of cellular networks. The ongoing call or data session is transferred from one base station to other while maintaining the services. This process is called Handover. Integration of different wireless technologies and thus forming a heterogeneous network has helped the users to have access to different radio access technologies. Providing seamless handovers and seamless mobility to the device is an important design issue in Heterogeneous networks. A vertical handover strategy accounts for seamless mobility across different access technologies. Many studies have been carried out to achieve improvement in handover strategies and many algorithms have been developed in this regard to perform smooth handovers across cell boundaries. Traditional algorithms consider only Received Signal Strength (RSS) from the target cell as the sole criterion for handover but that leads to unnecessary handovers because the device is handed over to a new cell as soon as it gets good signal strength. To reduce the unnecessary handovers, more than one condition should be considered for taking decision. This paper presents a handover strategy based on RSS and comparison of equivalent RSS of femtocell with macrocell which accomplishes the above goals. The paper is organized in the following manner: Section 2 presents a summary of literature related to the concept. Section 3 presents the steps of vertical handover procedure. The proposed algorithm and simulation results are described in Section 4 and 5 respectively, followed by conclusion in Section 6.

\section{RELATED WORK}

The diverse role of femtocell to provide improved quality and coverage has been described in paper [7]. This paper discusses that femtocell is an effective alternative to offload traffic frommacrocell. An overview of the mobility and handover management has been described in paper [8]. It also presents a handover strategy based on hysteresis margin and time to trigger which reduces the ping pong rate. The authors have shown in paper [9] that femtocell deployment can improve the network coverage indoors. It further presents a strategy to mitigate the interference between the two base stations. The bit error rate performance of macrousers and femto users under various load conditions has been studied in paper [10] and provides a useful deployment guide for heterogeneous networks. In paper [11], the authors have presented a handover decision algorithm based on interference, RSS and QoS level which reduces the unnecessary handovers. A comparative study of handover design parameters and salient features of various algorithms have been discussed in paper [12]. It has categorized various algorithms according to handover decision parameters and has provided an extensive survey of some very important handover algorithms. A cost 
function based algorithm for single macrocell and single femtocell has been presented in paper [13] which considers mobile's speed and traffic type to perform handover and reduces the handover probability for high speed users. Energy saving is always considered a very important issue in case of mobile devices. One such algorithm which aims to conserve the energy of mobile device is presented in paper [14] which takes into account the transmit power of cells, available bandwidth, the operating frequency and the interference level for performing handover. This algorithm is able to connect the mobile device to a cell which consumes minimum energy of device. A pure RSS based handover algorithm based on the comparison of RSS of source and target cells is presented in paper [15]. This algorithm improves the SINR performance at the device's side but accounts for more number of handovers due to absence of handover margin. A handover decision mechanism based on Radial Basis function (RBF) of neural networks is proposed in paper [16]. A handover optimization technique based on mobility prediction is presented in paper [17], which achieves good results in terms of reduced ping pong effect and increased throughput. The authors have proposed a method to deploy the Relay stations in cellular networks in paper [18] which addresses the issue of complete coverage and handover management.An adaptive vertical handoff algorithm based on compensating time is proposed in paper [19] which reduces the influence of shadow fading on RSS and provides for more effective and accurate handoff decisions. It further reduced the ping-pong effect and interruption probability.

\section{VERTICAL HANDOVER PROCEDURE}

Performing a handover within the same type of access networks is called horizontal handover, whereas performing handover in different types of access networks is defined as vertical handover. A vertical handover is performedin a heterogeneous environment, where more than one kind of networks are integrated together. Since, both have a different access technologies, when a user moves from one cell to another, the connection between base stations is also moved.The femtocell networks are cheap and easy to install but have limited coverage while macrocell networks cover a wide area but can only be installed by the network provider. The handover between macrocell and femtocell must be seamless i.e. the user should not observe disruption in any service, video, VOIP or data traffic. Two parameters affecting seamless handover are packet loss ratio and handover latency. Minimizing both of these parameters, leads to qualityhandover.

Figure 2 shows a heterogeneous network where macrocells are integrated with femtocells [20].

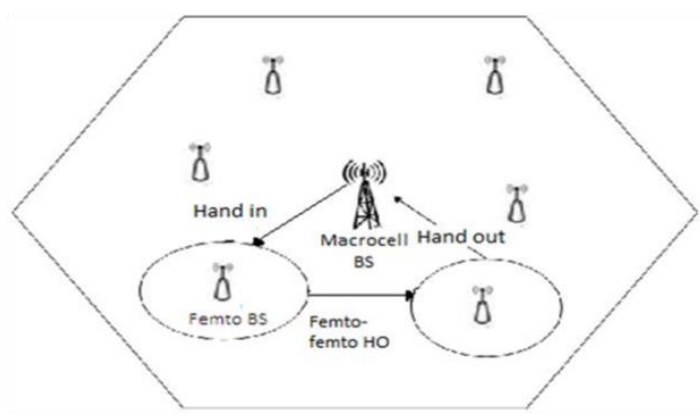

Figure 2. Integrated Macrocell Femtocell Networks

The handovers in such integrated networks are broadly classified as [21]:

a. Hand-in: These are the handovers from macrocell to femtocell and are a difficult task as the two have different backhaul rates and deployment of hundreds of femtocell a macrocell leads to a lot of options to choose from to perform a handover. When a mobile receives a signal from a femto base station, the report is sent to macro base station. The macro base station, along with the neighboring femto base stations prepares a neighboring cell list to choose an appropriate target cell. From the neighbor cell list the mobile device chooses the target femto base station based on signal levels received. The mobile device sends the request for handover to macro base station which forwards the request to femto base station through core network and femto gateway. The femto base station performs the necessary authorization checks and matches the interference levels and then replies to handover request. A new connection is established between femto gateway and femto base station. The mobile device now establishes a link to femto base station after a physical channel reconfiguration is performed and is disconnected from macro base station. The mobile device then sends a handover completion message to 
macro base station and coordinates with femto base station. The complete call flow procedure is shown in Figure 3.

b. Hand-out: These are the handovers from femtocell to macrocell and the decision is relatively easy as the target macrocell is always one. Here, the mobile device sends the report to connected femto base station when the signal goes down. A neighbor cell list is prepared by the femto and macro base stations and decision to perform handover to macrocell is taken. The mobile device sends a handover request to macro base station through femto gateway and radio network controller. In the similar way radio link is setup and physical channel reconfiguration is performed and mobile device gets connected to macro base station.

c. Femto-femto handovers: The handovers which are performed when a mobile device moves out of boundary of one femtocell and enters into a boundary of another femtocell, then femto to femto handover takes place. The decision is significant as the mobile device should preferably be connected to femtocell if it is getting required signal strength from both macrocell and femtocell. As the source femto signal goes weak, the mobile device sends a report to the connected femto base station. A neighbor cell list is prepared by the connected femto base station and macro base station. The mobile device and the femto base station take the decision to perform handover. The source femto base station sends a handover request to target femto base station through femto gateway. A new link is established between the femto gateway and target femto base station. The mobile device now sends the data through new femto base station.

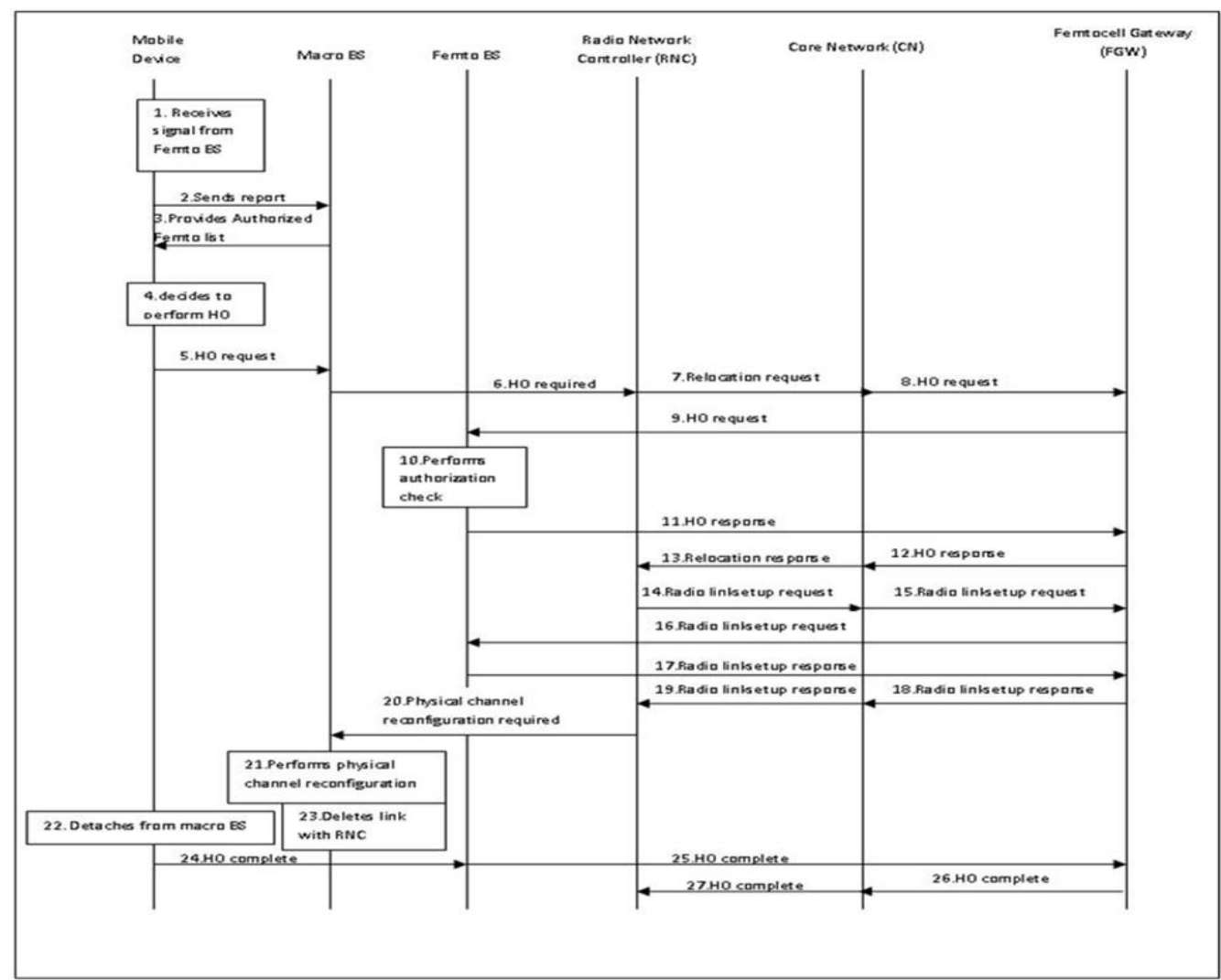

Figure 3. Call Flow Procedure for Handover from Macrocell to femtocell

\section{PROPOSED ALGORITHM}

This Section introduces a simple and efficient RSS based handover algorithm. As discussed in Section 2, most of the algorithms for handover in heterogeneous networks consider RSS as one of the metric. For performance comparison the traditional algorithm based on RSS and threshold value for RSS has been considered. The handover decision criteria for traditional algorithmas given in [22] can be expressed as

$$
\left(R S S_{t}>R S S_{s}\right) \text { and }\left(R S S_{s}<R S S_{t h}\right)
$$


Where $\mathrm{RSS}_{\mathrm{t}}$ and $\mathrm{RSS}_{\mathrm{S}}$ are the RSS of target and source cell respectively, $\mathrm{RSS}_{\mathrm{th}}$ is the threshold value for RSS. When the RSS of source cell falls below a threshold value, a target cell with RSS greater than the source is searched and a handover is performed. The algorithm leads to more number ofhandovers because even though the RSS from the target is higher by a small amount, the handover is performed.This causes the deterioration in RSS after a short interval and again a target cell is searched for handover leading to ping pong effect. To overcome the problem, additional parameters like speed, available bandwidth or Signal to Interference Noise ratio etc. may be considered to take the decision of handover. However, a combination of many different parameters increases the complexity of the algorithm. This further results in delayed handover process as femtocells have very low computational capability and the process of handover decision and execution is time consuming. Therefore, an RSS based algorithmis desired for its simplicity and reduction in unnecessary handovers.

Keeping in view the limitations discussed above, a handover algorithm based on equivalent RSS and handover margin is proposed. Sub Sections 4.1 and 4.2 present the calculation of effective RSS and handover margin respectively.

\subsection{Equivalent RSS}

There is a huge difference between the transmit powers of femtocell and macrocell i.e. $20 \mathrm{dBm}$ and $43 \mathrm{dBm}$ respectively. Therefore, the received power from femtocell, $\mathrm{RSS}_{\mathrm{f}}$ and received power from macrocell, $\mathrm{RSS}_{\mathrm{m}}$ cannot be compared directly. The equivalent RSS is calculated when RSS of femtocell and macrocell are compared. The proposed algorithm is based upon equivalent RSS of femtocell i.e. $\mathrm{RSS}_{\mathrm{fe}}$. The $\mathrm{RSS}_{\mathrm{fe}}$ would make the received signal strength from the two cells comparable. To calculate $\mathrm{RSS}_{\mathrm{fe}}$, some factor of $\mathrm{RSS}_{\mathrm{m}}$ is added to $\mathrm{RSS}_{\mathrm{f}}$ which is percentangeratio of Normalized transmitted power with respect to the distance between the two base stations. The distance between the two base stations is included to ensure that if the femtocell is located in the boundary region of macrocell, the mobile device always has the higher probability of being connected to the femtocell. The farther the femto base station from macro base station more is the value of $\mathrm{RSS}_{\mathrm{fe}}$ and the device has more probability of being connected to femto base station. This fact helps to optimize the performance as a very weak signal received from macro base station, at the boundary, leads to increased packet loss. Mathematically $\mathrm{RSS}_{\mathrm{fe}}$ is denoted as

$$
R S S_{f e}=R S S_{f}+\left(\frac{N T_{x}}{d}\right) * R S S_{m}
$$

where $\mathrm{d}$ is the distance, in meters, between the macro base station and femto base station and $\mathrm{NT}_{\mathrm{x}}$ is the Normalized Transmit power of a cell.

The Normalized transmit power is expressed as

$$
N T_{x}=\frac{T x_{m}-T x_{f}}{T x_{f}+T x_{m}}
$$

where $\mathrm{Tx}_{\mathrm{m}}$ and $\mathrm{Tx}_{\mathrm{f}}$ represent the transmit powers of macrocell and femtocell respectively.

\subsection{Handover Margin}

The handover is initiated when the target cell is better than the source cell by a certain margin. This reduces the unnecessary handovers and ping pong effect. The margin, $\delta$ calculated dynamically in the proposed algorithm is as given in [23]

$$
\delta=\max \left\{\delta \max \left\{1-\left(\frac{\text { Distance }}{\text { Radius }}\right)^{4}\right\}, 0\right\}
$$

where Radius is the radius of serving cell and Distance is the distance between mobile device and serving base station. $\delta_{\max }$ is the maximum value, which is calculated by taking difference between power received at the boundary of a cell and at a position closest to the base station.

The Equation 4 shows that if the device is near to the base station, the value of margin is more. Therefore, the RSS of target cell should be greater than the RSS of source cell by a significant amount for a handover to be initiated. This helps to reduce the number of handovers. On the other hand, if the device is far from the base station, the value of margin is less i.e. even a slightly higher RSS from the target cell leads to a handover. This helps to maintain a proper connection of mobile device to base station and thus leads to efficient packet delivery. 


\subsection{Algorithm}

Using Equations 2 and 4, the Hand In and Hand Out handover cases are discussed.

Case I: When the device is connected to macrocell: When the device is initially connected to macrocell and detects a signal from a femtocell, the RSS from all femtocells is checked. The equivalent RSS, RSS $\mathrm{fe}_{\mathrm{fe}}$ is calculated for each femtocell. A report consisting $\mathrm{RSS}_{\mathrm{f}}$ and $\mathrm{RSS}_{\mathrm{fe}}$ is sent to the macro base station. The macro base station prepares a neighbor cell list to choose the appropriate target cell. A handover to femtocell is performed if RSS is greater than the threshold RSS of femtocell. Simultaneously, it also compares $\mathrm{RSS}_{\mathrm{fe}}$ to $\mathrm{RSS}_{\mathrm{m}}$ and handovers the mobile device to femtocell if any of the two conditions are met. Otherwise, the device stays connected to macrocell.

$$
\left(R S S_{f} \geq R S S_{f t h}\right) \text { or }\left(R S S_{f e} \geq\left(R S S_{m}+\delta\right)\right)
$$

where $\mathrm{RSS}_{\mathrm{fth}}$ is the threshold value of femtocell and $\delta$ is the margin.

Case II: When the device is connected to femtocell: When the device is connected to femtocell, the signal from femtocell is constantly checked. If the signal diminishes beyond the threshold value of femtocell, $\mathrm{RSS}_{\text {th }}$, a report is sent by the mobile device to the femto base station. The femtocell base station prepares a neighbor cell list consisting of information about all the femtocells having higher RSS by a certain margin. If no femtocell with better RSS is found then $\mathrm{RSS}_{\mathrm{fe}}$ is calculated. The handover to macrocell is performed if $\mathrm{RSS}_{\mathrm{m}}$ is better than $\mathrm{RSS}_{\mathrm{fe}}$ by a certain margin. Otherwise, no handover is performed.

$$
\left(R S S_{f} \leq R S S_{f t h}\right) \text { and }\left(R S S_{m} \geq\left(R S S_{f e}+\delta\right)\right)
$$

The Margincalculation is given in Equation 4.

The Equations 5 and 6 and the Hand In and Hand Out procedure discussed above show that a device is always preferably connected to femtocell whenever the appropriate signal from femtocell is available. The working of proposed algorithm is shown in the flowchart given in Figure 4.

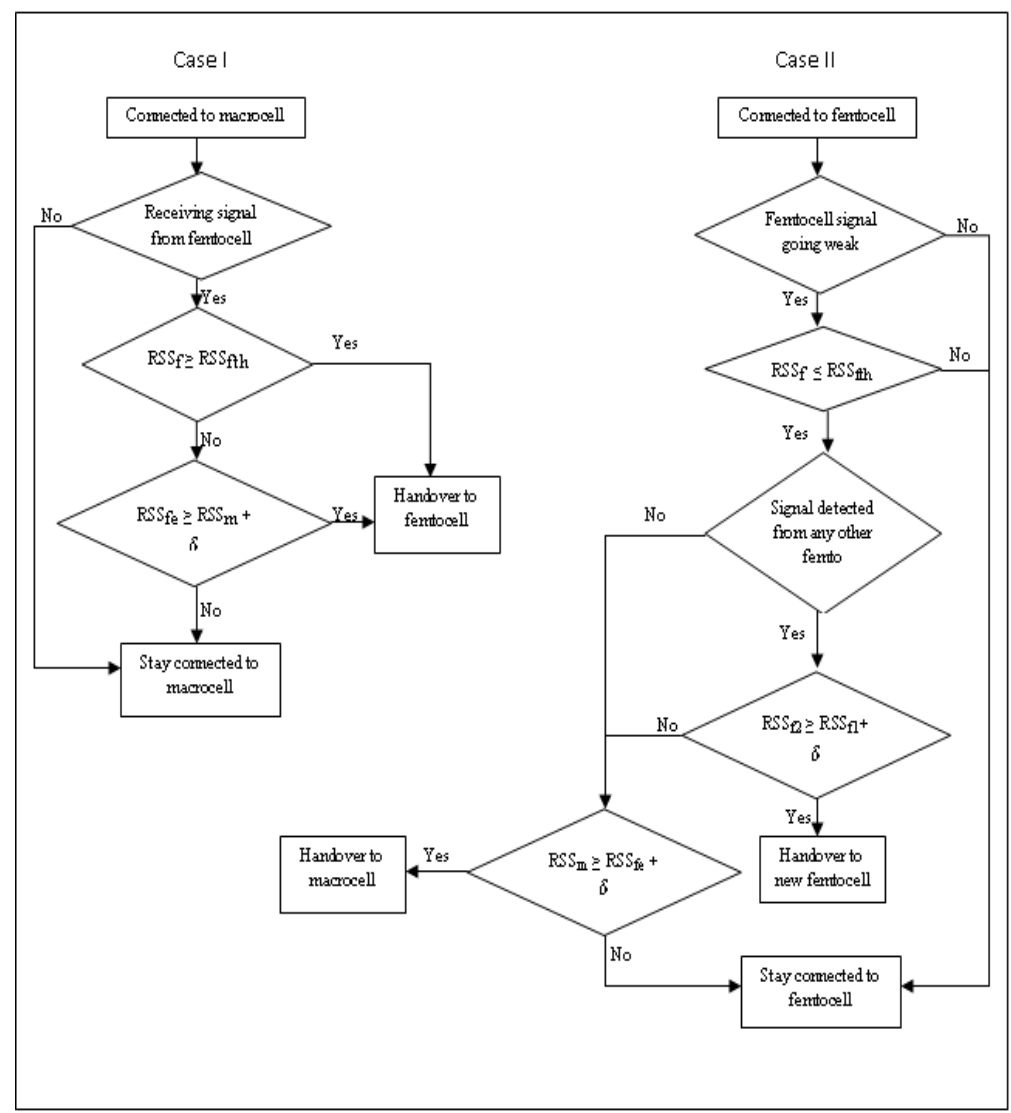

Figure 4. Flowchart of the proposed algorithm 
The key characteristics of the proposed algorithm are summarized below:

a. It considers the threshold RSS of femtocell for both hand-in and hand-out. It allows the mobile device to remain connected to femtocell whenever the signal from femtocell is available, so as to offload macro.

b. It calculates the dynamic value of margin based on the distance between mobile device and base station.It helps to take the handover decision at correct time and reduces unnecessary handovers.

c. It includes an additional condition of comparing the equivalent RSS of femtocell $\left(\mathrm{RSS}_{\mathrm{fe}}\right)$ with that of macrocell.

\section{SIMULATION RESULTS AND ANALYSIS}

The proposed algorithm is implemented for overlaid femto macrocellular networks. The simulation results are compared with traditional RSS based algorithm. The simulation has been carried out in LTE-Sim simulator. It is an open source simulator in which a scenario consisting of a single macrocell with overlaid femtocells has been created. The users are randomly placed in the macrocell near the femtocells. Some assumptions made in the algorithm are mentioned below:

a. When a macrocell is overlaid with femtocells, the mobile device is preferably connected to femtocell as soon as it gets receivedsignal strength from femtocell. This is required so that traffic can be diverted to femtocell whenever possible and macrocell is offloaded.

b. The mobile devices are placed near the femto base station so that the devices get good signal strength from femto base station. This is required so that handover to femtocell is performed within the stipulated simulation time.

c. Two speeds have been considered $30 \mathrm{kmph}$ and $120 \mathrm{kmph}$ corresponding to slow and fast moving vehicles.

Table 1. Simulation Parameters

\begin{tabular}{|c|c|c|}
\hline Parameter & Macrocell & Femtocell \\
\hline Radius & $1 \mathrm{Km}$ & $30 \mathrm{~m}$ \\
\hline Transmission power & $43 \mathrm{dBm}$ & $20 \mathrm{dBm}$ \\
\hline Threshold power & NA & $-80 \mathrm{dBm}$ \\
\hline Path loss Model & $\begin{array}{c}128.1+\left(37.6 * \log _{10}(\text { dista }\right. \\
\text { nce* } 0.001))\end{array}$ & $\begin{array}{l}\mathrm{A}^{*} \log _{10}(\text { distance })+\mathrm{B}+\mathrm{C} \log _{10}(\mathrm{fc} / 5) \\
\text { where } \mathrm{A}=20, \mathrm{~B}=46.4 \text { and } \mathrm{C}=20 \text { for } \operatorname{LOS} \text { transmission and } \\
\mathrm{A}=18.7, \mathrm{~B}=46.8 \text { and } \mathrm{C}=20 \text { for NLOS transmission }\end{array}$ \\
\hline Bandwidth & $20 \mathrm{MHz}$ & $20 \mathrm{MHz}$ \\
\hline Number of cells & 1 & 60 \\
\hline Number of users & & $10-50$ \\
\hline Simulation time & & $120 \mathrm{~s}$ \\
\hline Speed of users & & $30 \mathrm{kmph}, 120 \mathrm{kmph}$ \\
\hline
\end{tabular}

Based on the assumptions and simulation parameters mentioned in Table 1, the simulation is run for the integrated macro femtocellular networks. The number of handovers and Packet Loss Ratio in both traditional and proposed algorithm are compared and analyzed. The simulation parameters considered in the paper are given in Table 1.

Number of Handovers: The number of handovers with increase in number of users is depicted in Figure 5(a) and Figure 5(b). The results show that the number of handovers performed by the users is much less in proposed algorithm as compared to the traditional algorithm. This is because of the dynamic handover criteria chosen in the proposed algorithm. The Margin, $\delta$ is calculated based on the distance of mobile device from base station. Less is the distance from source base station more will be the margin. This ensures that mobile devices near the source base station need sufficiently high signal strength from target base station, in order for handover to be performed. When the vehicles move at a high speed, the position of device is changed rapidly and more handovers are performed showing ping pong effect. But, in the proposed algorithm, the number of handovers is comparable for both high speed and slow moving devices, as evident from Figure 5(b). This is because of the delaying the handover till the significant signal strength is available from the target base station. The proposed algorithm shows greater reduction in handovers at high speed.

Percentage reduction in handovers is given as

$$
H O_{\text {reduction }}=\left(\sum_{i=10}^{50} H_{T}-\sum_{I=10}^{50} H_{P}\right) / \sum_{i=10}^{50} H_{T}
$$


where $\mathrm{H}_{\mathrm{T}}$ is the handovers in traditional algorithm and $\mathrm{H}_{\mathrm{P}}$ is the handovers in proposed algorithm for 10 to 50 users.

Using above Equation and the values plotted in graph, an average $36.78 \%$ reduction in unnecessary handovers for users moving at a speed of $30 \mathrm{kmph}$ and $61.85 \%$ reduction for users moving at a speed of $120 \mathrm{kmph}$ is obtained as shown in Figure 5(a) and Figure 5(b) respectively.

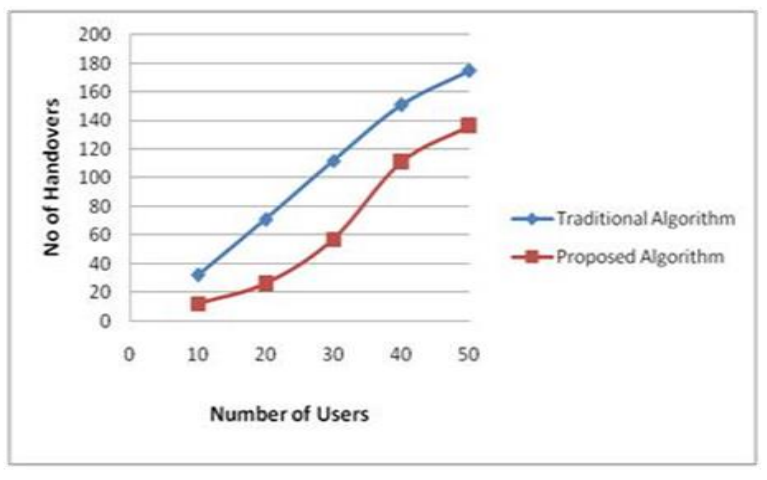

(a)

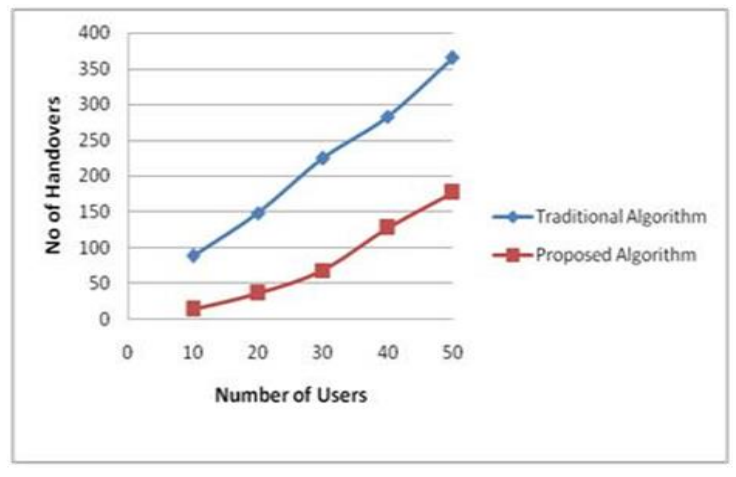

(b)

Figure 5. (a) No of Handovers vs. No of users at $30 \mathrm{kmph}$, (b) No of Handovers vs. No of users at $120 \mathrm{kmph}$

Packet Loss Ratio (PLR): Packet Loss Ratio (PLR) is defined as the ratio of packet lost to the total packet sent during a simulation expressed by Equation 8.

$$
P L R=\left(\sum_{i=1}^{n} P r_{i}-\sum_{i=1}^{n} P s_{i}\right) / \sum_{i=1}^{n} P s_{i}
$$

where $\mathrm{Ps}_{\mathrm{i}}$ is the packet sent and $\mathrm{Pr}_{\mathrm{i}}$ is the packet received. The variation of PLR with increase in the number of usersis shown in Figure 6(a) and Figure 6(b). It shows that Packet Loss Ratio is much less in case of proposed algorithm as compared to the traditional algorithm. The decrease in PLR is due to the fact that the handover decision is more accurate and is a function of equivalent RSS and margin.

$$
\text { Handover Decision }=f\left(\mathrm{RSS}_{\mathrm{fe}}, \text { margin }\right)
$$

The device is made to spend more time connected to the femtocell base station. The longer the connection of device is maintained with the base station, the channel is used for sending and receiving the data packets instead of transmitting the control packets. The timely and correct handover decision would reduce the packet lost. In light of the above cited reasons, the proposed algorithm shows improvement for both slow vehicles moving at a speed of $30 \mathrm{kmph}$ and fast vehicles moving at a speed of $120 \mathrm{kmph}$ as shown in Figure 6(a) and Figure 6(b) respectively.

The traditional algorithm shows a very high value for packet loss ratio, with the increase in number of users. The average percentage decrease in PLR is defined as

$$
P L R_{\text {reduction }}=\left(\sum_{i=10}^{50} P L R_{T}-\sum_{I=10}^{50} P L R_{P}\right) / \sum_{i=10}^{50} P L R_{T}
$$

where $\mathrm{PLR}_{\mathrm{T}}$ is the packet loss ratio in traditional algorithm and $\mathrm{PLR}_{\mathrm{P}}$ is the packet loss ratio in proposed algorithm for 10 to 50 users.

Using above Equation and the values plotted in graph, there is an average 23.03\% reduction in PLR when users are moving at a speed of $30 \mathrm{kmph}$ and $55.27 \%$ reduction when users are moving at a speed of $120 \mathrm{kmph}$. 
A mobile device moving at a fast speed has a tendency of performing handovers more frequently and thus leading to unnecessary handovers. The proposed algorithm shows a significant reduction in number of unnecessary handovers for both slow and fast moving vehicles.

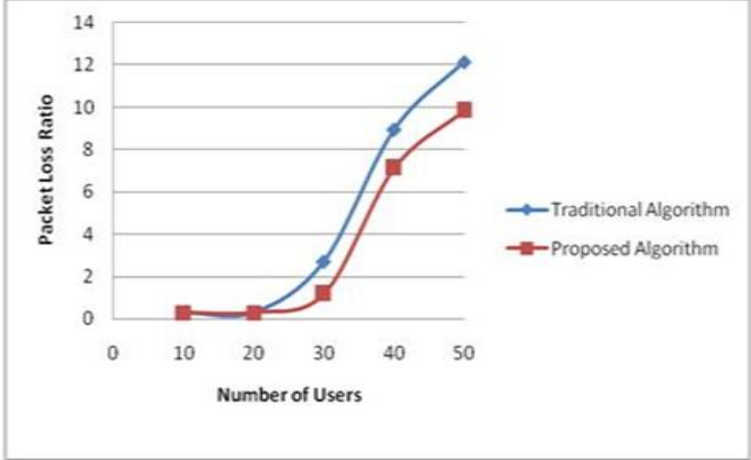

(a)



(b)

Figure 6. (a) Packet Loss Ratio vs No of users at 30kmph, (b) Packet Loss Ratio vs No of users at $120 \mathrm{kmph}$

\section{CONCLUSION}

This paper presented a new handover algorithm which is applied when a mobile device enters or leaves the coverage area of femtocell. The proposed handover algorithm calculated the equivalent received signal strength along with dynamic margin for performing handover. The simulation results of proposed algorithm were compared with the traditional algorithm. The handover strategy showed improvement in two major performance parameters namely, reduction in unnecessary handovers and Packet Loss Ratio. The simulation results of the proposed algorithm werequantitatively analyzed and compared with traditional algorithm. The proposed handover strategy showed $55.27 \%$ and $23.03 \%$ reduction in packet loss ratio and $61.85 \%$ and $36.78 \%$ reduction in unnecessary handovers at a speed of $120 \mathrm{kmph}$ and $30 \mathrm{kmph}$ respectively. Reduction in packet loss ratio signified that the mobile device is having a better signal for longer period of time. Reduction in number of unnecessary handovers ensured better utilization of resources of the network. This algorithm allowed the mobile device to spend more time in femtocell and thus tries to offload macrocell. It performed better than conventional RSS based handover algorithm, both for slow and fast moving users.

\section{FUTURE SCOPE}

The analysis is carried out by simulating the scenario of overlaid macrocell and femtocell structure. Current algorithm shows better results in terms of reduced unnecessary handovers and reduced packet loss ratio. The work could be further extended by combining RSS with some other parameters for performing handovers, which can help further to achieve better results.

\section{REFERENCES}

[1] Baig, Imran, and Varun Jeoti, "A novel precoding based hybrid MC/SC radio access system for PAPR reduction in layered OFDMA of LTE-Advanced”, IAENG International Journal of Computer Science, vol. 38, pp. 314-319, 2011.

[2] 3GPP TS 36.300 V9.5.0 (2010-09) "Evolved Universal Terrestrial Radio Access (E-UTRA) and Evolved Universal Terrestrial Radio AccessNetwork (E-UTRAN) overall description”, Stage 2 (Release 9).

[3] Harri Holma, Antti Toskala, "LTE-The UMTS long Term Evolution: From Theory to Practice", John Wiley \& Sons Ltd, 2009.

[4] Tsung-Hui Chuang, Guan-Hong Chen, Meng-Hsun Tsai, Chun-Lung Lin," Alleviating Interference through Cognitive Radio for LTEAdvanced Network", International Journal of Electrical and Computer Engineering, vol. $5,2015$.

[5] Jie Zhang, Guillaume de la Roche, Femtocells: Technologies and Deployment, John Wiley \& Sons, 2010.

[6] Sharma, Madhu J., and Victor CM Leung, "IP Multimedia subsystem authentication protocol in LTEheterogeneous networks", Human-Centric Computing and Information Sciences, vol. 2, pp. 1-19, 2012. 
[7] Ahmed, Afaz Uddin, Mohammad Tariqul Islam, and Mahamod Ismail, "A review on femtocell and its diverse interference mitigation techniques in heterogeneous network", Wireless Personal Communications, vol. 78, pp. 85106, 2014.

[8] Lim, Jaechan, and Daehyoung Hong, "Mobility and handover management for heterogeneous networks in LTEadvanced", Wireless personal communications, vol. 72, pp. 2901-2912, 2013.

[9] Shalaby, Mohamed, Mona Shokair, and Nagy Wadie Messiha, "System Design and Performance Analysis of LTE Cognitive Femtocells”, Wireless Personal Communications, vol. 85, pp. 2463-2483, 2015.

[10] Hussain, Fatima, Alagan Anpalagan, and Hyung Yun Kong, "Performance analysis of two dimensional spreading for ofcdm femtocell users overlaid with ofdm macrocell”, Wireless personal communications, vol. 72, pp. 137$158,2013$.

[11] Wu, Shih-Jung, "A new handover strategy between femtocell and macrocell for LTE-based network", in 4th International Conference on Ubi-Media Computing (U-Media)", IEEE, 2011.

[12] Xenakis, Dionysis, "Mobility management for femtocells in LTE-advanced: key aspects and survey of handover decision algorithms", Communications Surveys \& Tutorials, IEEE, vol. 16, pp. 64-91, 2014.

[13] D. Lee, G. Gil, D. Kim, "A Cost-Based Adaptive Handover Hysteresis Scheme to Minimize the Handover Failure Rate in 3GPP LTE System", EURASIP J. on Wireless Communication and Networks, vol. 6, 2010.

[14] Xenakis, Dionysis, Nikos Passas, and Christos Verikoukis, "An energy-centric handover decision algorithm for the integrated LTE macrocell-femtocell network”, Computer Communications, vol. 35, pp. 1684-1694, 2012.

[15] Yang, Gang, Xiaoyang Wang, and Xiaolu Chen. "Handover control for LTE femtocell networks." International Conference on Electronics, Communications and Control (ICECC), IEEE, 2011.

[16] Payal Mahajan, Kuldeep Singh, Hardeep Kaur, "Handover Decision Mechanism in Interworking Technologies Using Radial Basis Functions", IAES International Journal of Artificial Intelligence (IJ-AI), Vol 3 No 2, 2014

[17] Kim, Tae-Hyong, and Jae-Woo Kim, "Handover optimization with user mobility prediction for femtocell-based wireless networks", International Journal of Engineering and Technology (IJET), vol. 5, pp. 1829-1837, 2013.

[18] Ratheesh, R., and P. Vetrivelan, "Power Optimization Techniques for Next Generation Wireless Networks", International Journal of Engineering and Technology (IJET), vol. 8, pp. 247-257, 2016.

[19] Jin Ling, Zhang Hui, Yang Long-Xiang, Zhu Hong-Bo, "An Adaptive Vertical Handoff Algorithm Based on UMTS and WLAN", IAES TELKOMNIKA Indonesian Journal of Electrical Engineering, Vol 12 No 5, 2014, pp. 3943-3954.

[20] Suman Deswal, Anita Singhrova, "A Review of Handover Schemes in Overlaid Macro femto Cellular Networks", in International Conference on Wireless Networks, ICWN'14, July 2014, pp: 261-265, CSREA press, ISBN: 1-60132-278-X.

[21] Chowdhury, Mostafa Zaman, and Yeong Min Jang, "Handover management in high-dense femtocellular networks", EURASIP Journal on Wireless Communications and Networking, vol.1, pp. 1-21, 2013.

[22] Kassar, Meriem, Brigitte Kervella, and Guy Pujolle. "An overview of vertical handover decision strategies in heterogeneous wireless networks" Computer Communications, ELSEVIER, vol. 31, pp. 2607-2620, 2008.

[23] Becvar, Zdenek, and Pavel Mach, "Adaptive hysteresis margin for handover in femtocell networkks", in 6th International Conference on Wireless and Mobile Communications (ICWMC), IEEE, 2010.

[24] Bagubali, A., Prithiviraj, V., Mallick, P.S., \& Krishnan, K.V., "Handover Problemfor Integrating LTE with Femtocell Network", Journal of Theoretical \& Applied Information Technology, vol. 46, 2012.

\section{BIOGRAPHIES OF AUTHORS}

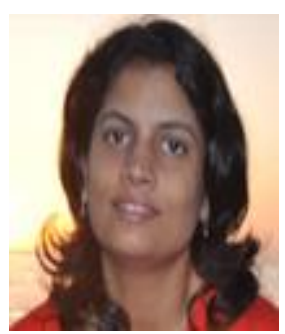

Suman Deswal is pursuing her Ph.D from DCR University of Science \& Technology, Murthal, India. She has completed her M.Tech (Computer Science \& Engg.) from Kurukshetra University, Kurukshetra, India and B.Tech (Computer Science \& Engg.) from CR State College of Engg., Murthal, India in 2009 and 1998 respectively. She possesses 14 years of teaching experience and is presently working as Assistant Professor in the department of Computer Science and Engg. at DCR University of Science and Technology, Murthal, India.

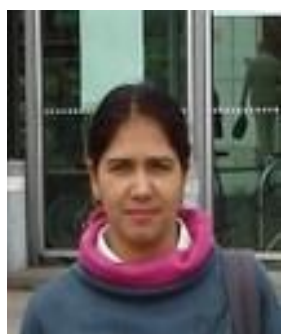

Anita Singhrova holds a Ph.D degree from GGS Indraprastha University, Delhi, India. She has completed M.E (Computer Science \& Engg.) from Punjab Engineering College, Chandigarh, India and B.Tech (Computer Science) from T.I.T\&S, Bhiwani, India 2006 and 1993 respectively. She has also been certified as Java Programmer by Sun Microsystems. She possesses seventeen years of teaching experience and is presently working as Professor in the department of Computer Science and Engg. at DCR University of Science and Technology, Murthal, India. 